\title{
CORRELACIÓN ENTRE LA ACELERACIÓN Y VELOCIDAD MÁXIMA DEL SUELO: APLICACIÓN EN EL ANÁLISIS DEL PELIGRO SÍSMICO
}

\author{
Miguel A. Jaimes $^{(1)}$, Eduardo Reinoso ${ }^{(1)}$, Mario Ordaz $^{(1)}$ y Cesar Arredondo ${ }^{(1)}$
}

\begin{abstract}
RESUMEN
Se propone una expresión que correlaciona los parámetros sísmicos de aceleración $\left(A_{\max }\right)$ y velocidad $\left(V_{\max }\right)$ máximas del suelo que son usados frecuentemente en la estimación de daño en tuberías enterradas, contenidos y elementos no estructurales. Esta expresión permite estimar el valor del parámetro $\left(V_{\max }\right)$ en función de $A_{\max }$, por lo que, de una ecuación de atenuación existente para $A_{\max } \mathrm{y}$ de un modelo de la actividad sísmica de la fuente, es posible realizar un análisis probabilista del peligro sísmico de tipo bivariado. Se presenta un ejemplo de la obtención del peligro sísmico bivariado para dos sitios de terreno firme.
\end{abstract}

\begin{abstract}
We propose an equation that correlates the seismic parameters of peak ground motion acceleration $\left(A_{\max }\right)$ and velocity $\left(V_{\max }\right)$ commonly used to estimate damage to pipelines, building contents and non structural elements. This equation allows us to estimate peak velocity $V_{\max }$ in terms of peak acceleration $A_{\max }$. This means that, from an existing attenuation relation for $A_{\max }$ and a source model, it is possible to carry out a bivariated probabilistic seismic hazard analysis. An example of the probabilistic hazard assessment is presented for two sites at hill zone.
\end{abstract}

\section{INTRODUCCIÓN}

Es frecuente que los análisis probabilistas de peligro sísmico se lleven a cabo caracterizando la intensidad sísmica con un solo parámetro, el que empíricamente mejor correlacione la intensidad del sismo con el daño. Sin embargo, es reconocido que es limitado describir con precisión un fenómeno complejo con un sólo parámetro, dado que inevitablemente una gran cantidad de información se pierde (Housner y Jennings, 1982). En edificaciones, por ejemplo, se usa principalmente la aceleración espectral que está asociada a las fuerzas inerciales impuestas a la estructura, y el desplazamiento; sin embargo, los daños en tuberías, contenidos y elementos no estructurales no están correlacionados solamente con estos parámetros de intensidad.

La intensidad se expresa en términos de una variable escalar, tal como los valores máximos de aceleración $\left(A_{\max }\right)$, velocidad $\left(V_{\max }\right)$ y desplazamiento $\left(D_{\max }\right)$ del movimiento. Estos máximos del movimiento del terreno al ser procesados figuran como los más aceptados en la caracterización de la

Artículo recibido el 3 de abril de 2008 y aprobado para su publicación el 15 de noviembre de 2009. Se aceptarán comentarios y/o discusiones hasta cinco meses después de su publicación

(1) Instituto de Ingeniería, UNAM, Ciudad Universitaria, 04510 México, D.F. mjaimest@iingen.unam.mx, ere@pumas.ii.unam.mx,mors@pumas.ii.unam.mx, carredondov@iingen.unam.mx 
demanda para contenidos (Ishiyama 1982 y 1984; Choi y Tung, 2002; Konstantinidis y Makris, 2003 y 2006; Al Abadi et al., 2006) y en sistemas no estructurales como tuberías enterradas (por ejemplo, Katayama et al., 1975; O'Rourke y Ayala, 1993; Pineda y Ordaz, 2004), las relaciones de fragilidad se expresan en términos de $V_{\max }$. Sin embargo, el uso aislado de estos parámetros puede llevar a una descripción inadecuada del daño (Riddell, 2007) debido a que $A_{\max }$ es un parámetro útil para sistemas rígidos, $V_{\max }$ es mejor para sistemas de frecuencia intermedia y $D_{\max }$ es mejor para sistemas flexibles.

En los últimos años se han propuesto parámetros que combinan relaciones de $A_{\max }$ y $V_{\max }$ que reflejan mejor la estimación de los daños en contenidos y elementos no estructurales. Algunos de estos son los siguientes:

- Los parámetros $V^{2}{ }_{\max } \mathrm{y} A_{\max }$ se correlacionan con el daño por deslizamiento en contenidos (Choi y Tung, 2002, Konstantinidis y Makris, 2003).

- La relación $A_{\max } / V_{\max }$ se adopta por Tso et al. (1992) como una medida característica del contenido de frecuencias de un movimiento.

- El índice $V^{2}{ }_{\max } / A_{\max }$ se ha usado como un indicador del daño en tuberías enterradas dada la estrecha relación de este parámetro con el desplazamiento máximo del suelo (Pineda, 2006).

- Los parámetros $V_{\max }^{2} / A_{\max }$ y $\left(V_{\max } / A_{\max }\right)^{2}$ se correlacionan con el volteo en contenidos desplantados en campo libre (Arredondo y Reinoso, 2008). El uso del primer parámetro se debe a que la ocurrencia del volteo está directamente relacionada a la capacidad de desplazamiento de un objeto, medida en su centro de gravedad respecto a los niveles de aceleración del mismo o en la superficie portante (Al Abadi et al., 2006). El segundo parámetro está relacionado con la frecuencia de vibración del movimiento; se ha observado que es muy importante en la respuesta dinámica de contenidos que podrían ser más vulnerables ante movimientos de baja frecuencia (Psycharis et al., 2002; Arredondo y Reinoso, 2008).

De lo anterior se desprende la necesidad de realizar un análisis probabilista del peligro sísmico (APPS) de tipo bivariado (asociado a dos parámetros sísmicos) para conocer la distribución de probabilidad conjunta de $A_{\max }$ y $V_{\max }$, por lo que es preciso contar con relaciones de atenuación que correspondan a ambos parámetros sísmicos y su posible correlación.

Desafortunadamente, no es común obtener relaciones de atenuación donde dadas la magnitud y la distancia se obtengan los valores de $A_{\max }$ y $V_{\max }$ de manera conjunta, así como la correlación entre ellas. En general, las leyes de atenuación sísmica se construyen para estimar $A_{\max }$ en función principalmente de la magnitud y distancia, lo que implica el cálculo de un conjunto de coeficientes a través de métodos de regresiones (p.e. Ordaz et al., 1989; Ambraseys, 2006; Abrahamson y Silva, 2008); pocas veces se construyen leyes de atenuación de $V_{\max }$ (p.e. García et al. 2005, Akkar y Bommer, 2007). En años recientes, se elaboró un proyecto denominado "Next Generation of Attenuation, NGA" para el occidente de los Estados Unidos. Como parte de este proyecto, varios autores estudiaron leyes de atenuación en términos de la velocidad (p.e. Boore y Atkinson, 2008; Campbell y Bozorgnia, 2008, Chiou y Youngs, 2008), además de la correlación que existe entre valores de aceleración del suelo y aceleración espectral entre distintos periodos y orientaciones (Baker y Jarayam, 2008).

En este trabajo se presenta una expresión aplicable para sitios en México que correlaciona los parámetros sísmicos de aceleración $\left(A_{\max }\right)$ y velocidad $\left(V_{\max }\right)$ máximas del suelo. Esta expresión permite estimar el valor del parámetro $V_{\max }$ en función de $A_{\max }$. A través de esta ecuación, de una ley de atenuación 
existente para $A_{\max }$ y de un modelo de la actividad sísmica de la fuente, es posible realizar un APPS de tipo bivariado, que es útil en la evaluación de pérdidas en contenidos y elementos no estructurales. Finalmente, se presenta un ejemplo de la obtención del peligro sísmico bivariado para dos sitios de terreno firme.

\section{SITIOS Y EVENTOS USADOS}

Para el cálculo de una expresión de la correlación entre la aceleración y velocidad máximas (implícitamente la estimación de $V_{\max }$ en función de $A_{\max }$ ) se revisaron y seleccionaron acelerogramas registrados dentro y fuera del valle de México. Los registros considerados son de sismos de subducción y de falla normal. Se tomaron solamente en cuenta las componentes horizontales, dado que el movimiento vertical es, especialmente para la Ciudad de México y estaciones distantes al foco, menos importantes desde el punto de vista ingenieril. Los sismos seleccionados tienen magnitudes de entre 5.0 y 8.1. De los registros de aceleración se leyó directamente el valor de $A_{\max }$. Para calcular $V_{\max }$ de los acelerogramas, se integra la historia de aceleración después de aplicar una corrección por línea base, sumando la constante necesaria para obtener un valor medio igual a cero considerando todos los puntos y un filtro pasabanda entre 0.1 y $10 \mathrm{~Hz}$ (Oppenheim y Schafer, 1975) para evitar la inclusión de ruido de alta frecuencia en los registros. La fig. 1 muestra algunos de los eventos sísmicos importantes usados, con fecha, magnitud y localización epicentral (círculos y cuadrados corresponden a sismos de subducción y falla normal, respectivamente). Asimismo, se indican las estaciones acelerométricas (triángulos) desplantados en sitios de roca en campo libre usadas en este trabajo y que se encuentran fuera del valle de México.

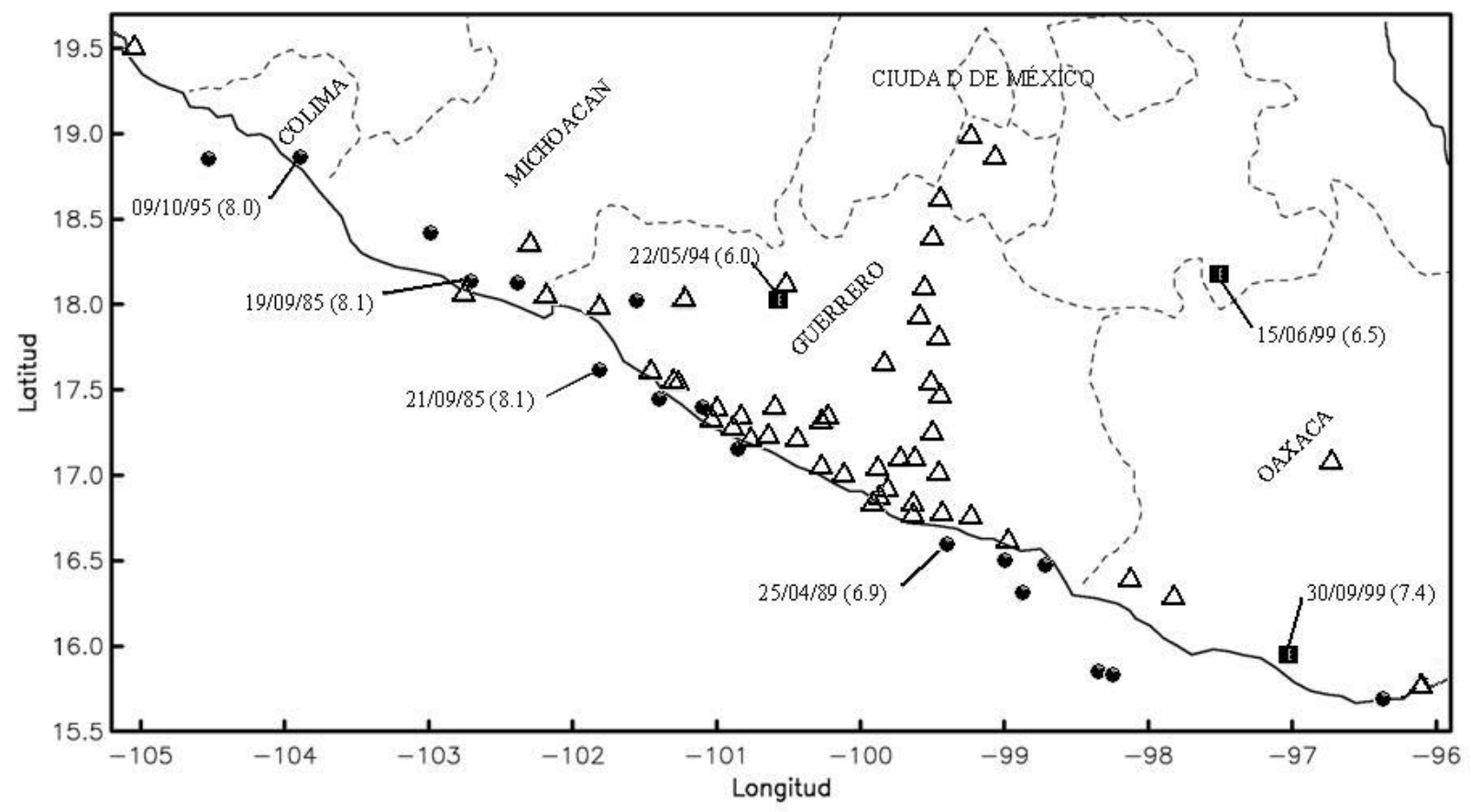

Figura 1. Localización de estaciones (triángulos) y epicentros de los eventos usados en este estudio; con círculos y cuadrados los sismos de subducción y falla normal, respectivamente

En la fig. 2 se muestra las estaciones acelerométricas del valle de México usadas. Las tres principales zonas geotécnicas están indicadas: (I) Zona de lomas, localizada en las partes altas del valle, formada por rocas volcánicas, suelos pocos compresibles y alta resistencia, (II) Zona de transición, con 
características mixtas de zona de lomas y lago, (III) Zona de lago, consiste de depósitos aluviales, lacustres y de arcilla altamente compresible con altos contenidos de agua.

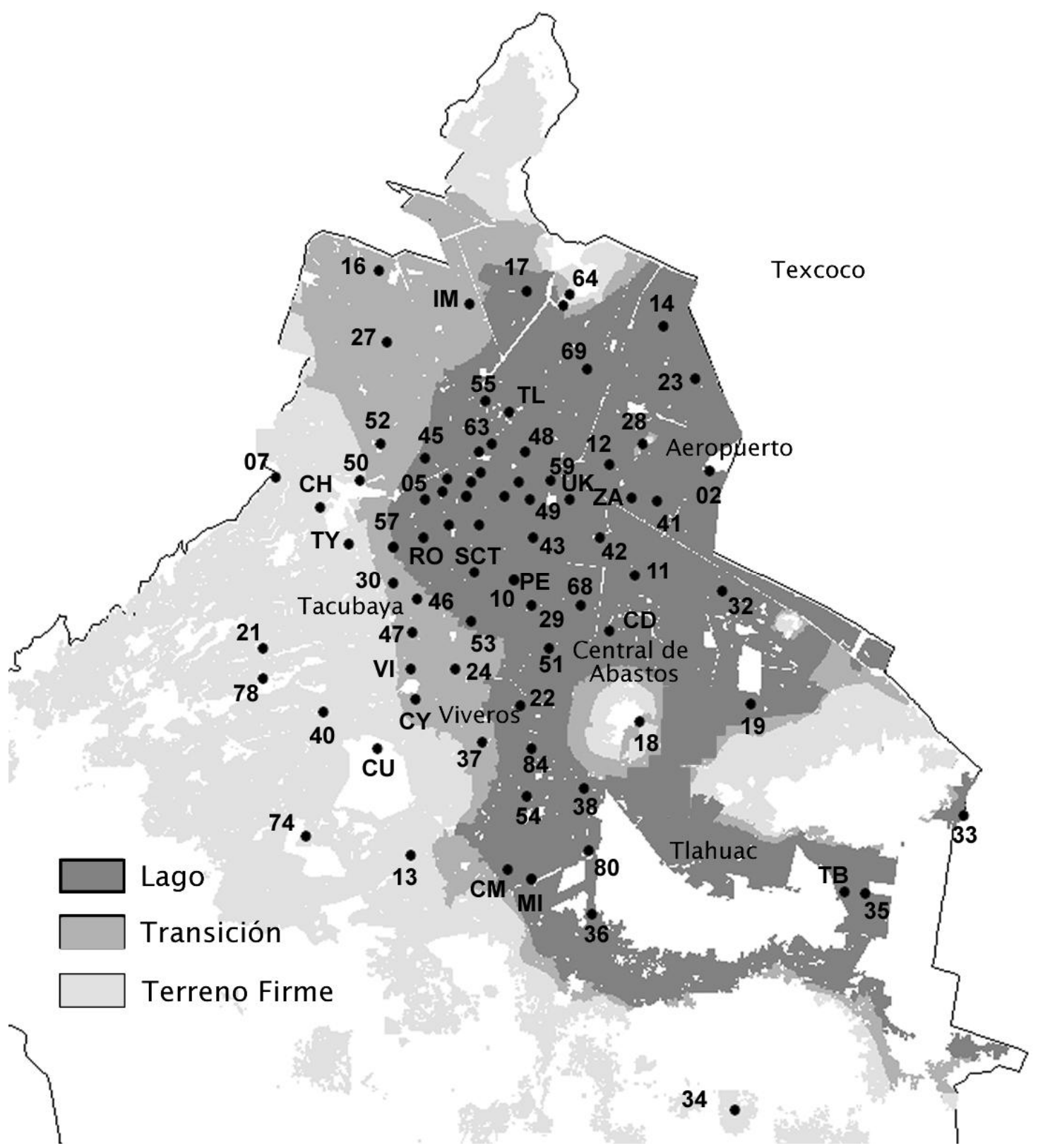

Figura 2. Estaciones acelerométricas del valle de México usadas. Se indican las zonas geotécnicas y algunos sitios de interés. 


\section{EL PARAMETRO $\omega$}

Si el movimiento sísmico del suelo fuera armónico, entonces $A_{\max }$ y $V_{\max }$ estarían perfectamente correlacionadas y se cumpliría que

$V_{\max }=\frac{A_{\max }}{\omega}$

donde $\omega$ es la frecuencia circular natural del movimiento.

Puede notarse que una deducción empírica de $\omega$ nos llevaría a una estimación simple de la correlación entre ambos parámetros. Algunos trabajos previos presentan valores de $\omega$ en diferentes tipos de suelos calculados como valores medios de los obtenidos a partir de movimientos registrados. Es el caso de estudios como los de Newmark y Hall (1982) recomiendan para condiciones de roca y suelo, en California, usar valores de $\omega=10.8$ y $8.0 \mathrm{rad} / \mathrm{s}$, respectivamente. Santa-Cruz et al. (2000) encontraron valores promedio para la ciudad de Acapulco de $28 \mathrm{rad} / \mathrm{s}$, y valores de 3.5 y $3.0 \mathrm{rad} / \mathrm{s}$ para terreno firme y blando de la ciudad de México, respectivamente. Sin embargo, como se mostrará más adelante, estos valores promedio pueden calcularse de manera más precisa considerando las condiciones geotécnicas e intensidades sísmicas que se presentan en el sitio.

\section{Cálculo del parámetro $\omega$ de un movimiento sísmico}

Encontrar el parámetro $\omega$ de un movimiento sísmico no es sencillo puesto que según el método que se utilice se obtienen valores distintos. Arredondo y Reinoso (2008) discuten la influencia de seleccionar diferentes maneras de calcular $\omega$, entre ellas: la frecuencia asociada al valor pico del espectro de amplitudes de Fourier, la frecuencia asociada a los máximos del espectro de velocidad y aceleración, la expresión de Fajfar et al. (1992), la frecuencia correspondiente al punto de intersección entre las regiones espectrales de aceleración constante y la de velocidad constante en un espectro de velocidad. Rathje et al. (1998) describe varios parámetros para estimar $\omega$ y desarrolla relaciones para predecir ésta en función de la magnitud, distancia y condiciones de sitio. Rathje et al. (2004) reexamina los parámetros para estimar $\omega$ encontrando que la siguiente ecuación es la que mejor proporciona un parámetro escalar debido a que representa las amplitudes de las ondas armónicas y la forma del espectro de amplitudes de Fourier e implícitamente la energía del sismo

$\omega=\frac{\int|A(w)|^{2} d w}{\int \frac{|A(w)|}{w} d w}$

donde $A(w)$ es el espectro de amplitudes de Fourier y $w$ es la frecuencia. En nuestro caso, para los fines de la correlación que se busca entre $A_{\max }$ y $V_{\max }$, utilizaremos la ec. 2.

En la fig. 3 se presenta para cuatro sitios el espectro de amplitudes de Fourier (EAF) correspondiente al sismo del 19 de septiembre de 1985, así como el valor del parámetro escalar $\omega$ resultado de la ec. 2. Nótese que el valor de $\omega$ difiere del valor pico del EAF e inclusive podría encontrarse en un valle del EAF; sin embargo, como se presentará más adelante, este es un parámetro adecuado de correlación entre $A_{\max }$ y $V_{\max }$ que proporciona la mejor caracterización del contenido de frecuencias, por lo que la ec. 2 será usada para estimar $\omega$ a lo largo del artículo. 


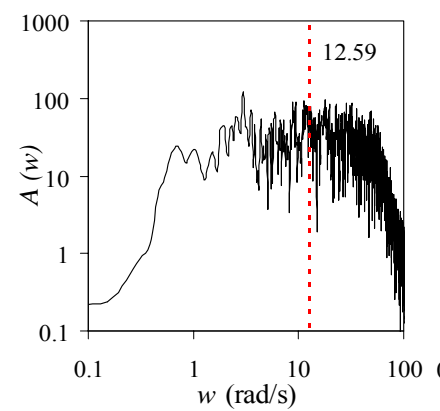

(a) Caleta de Campos

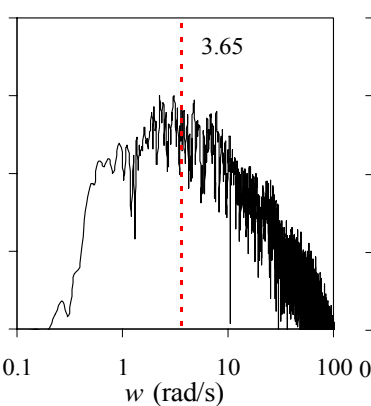

(b) $\mathrm{CU}$

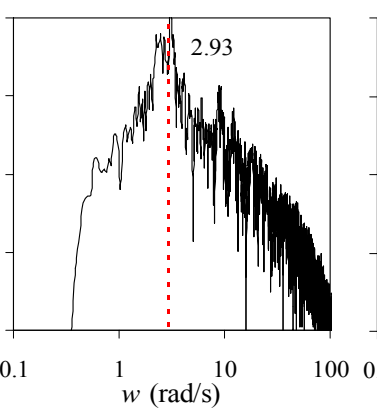

(c) SCT

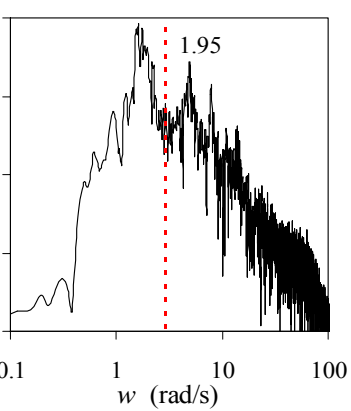

(d) CD

Figura 3. Espectros de amplitudes de Fourier del sismo del 19 de septiembre de 1985 para cuatro sitios en México. Se indica el valor del parámetro $\omega$ resultado de la ec. 2

\section{ESTIMACIÓN DE UNA EXPRESIÓN DE $\omega$ PARA EL VALLE DE MÉXICO}

Para sitios en el valle de México se obtuvieron las intensidades pico $\left(A_{\max }\right.$ y $\left.V_{\max }\right)$ y el parámetro $\omega$ para 452, 504 y 2802 movimientos sísmicos de sitios en terreno firme, transición y lago, de los cuales 2332 y 1426 corresponden a sismos de subducción y falla normal, respectivamente. La separación de movimientos sísmicos de acuerdo al origen del sismo es debido a que se observaron diferencias en los resultados.

\section{$\omega$ en función de la intensidad}

Se han observado diferencias importantes en el contenido de frecuencias ocurridos a distancias epicentrales aproximadamente iguales pero con diferente magnitud sísmica (Reinoso y Ordaz, 1999), por lo que es posible esperar una clara dependencia del parámetro $\omega$ con la magnitud y por lo tanto con la intensidad sísmica. En la fig. 4 se muestra la relación entre $A_{\max }$ y $\omega$ obtenida para tres sitios (CU, SCT y CD) ante sismos de subducción (círculos) y falla normal (cuadrados), con sus respectivas tendencias centrales mediante una línea continua y discontinua, respectivamente. Se puede observar cómo $\omega$ decrece de manera inversamente proporcional a la intensidad $\left(A_{\max }\right)$ en los tres sitios y para ambos tipos de sismos debido a que los eventos grandes (implícitamente mayor $A_{\max }$ ) son movimientos de baja frecuencia (Reinoso y Ordaz, 1999). Nótese que los sismos de falla normal presentan mayor contenido de alta frecuencia con respecto a los sismos de subducción para bajas intensidades.

\section{$\omega$ en función del periodo del sitio}

Debido a la naturaleza de la composición geotécnica del valle de México es posible esperar que el parámetro escalar $\omega$ esté caracterizado casi exclusivamente por los efectos de sitio, haciéndolo independiente del contenido de frecuencias propio de la fuente y atenuación sísmica. Aún en sitios en la ciudad de México considerados como terreno firme, es posible esperar movimientos sísmicos de banda relativamente angosta, ya que existe una diferencia importante entre las rigideces de los basamentos profundos y la roca en superficie, provocando amplificaciones anómalas en frecuencias alrededor de 0.5 $\mathrm{Hz} \approx 3.14 \mathrm{rad} / \mathrm{s}$ (Ordaz y Singh, 1992). En la fig. 5 se presenta la relación del periodo del suelo $T_{\mathrm{s}}$ y el parámetro $\omega$ (calculado con la ec. 2) para los tres sitios mencionados $\mathrm{CU}, \mathrm{SCT}$ y $\mathrm{CD}$, ante sismos de subducción (cuadrados) y falla normal (triángulos), con sus respectivas tendencias centrales mediante una línea gruesa discontinua y continua, respectivamente; con línea delgada gris se muestran los valores que corresponden a la frecuencia del suelo $\left(\omega_{\mathrm{s}}=2 \pi / T_{\mathrm{s}}\right)$. 


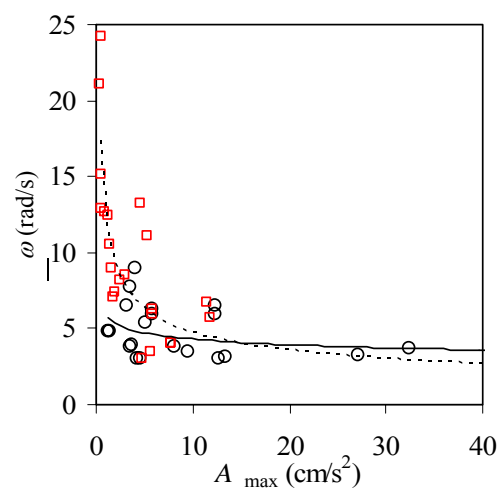

(a)

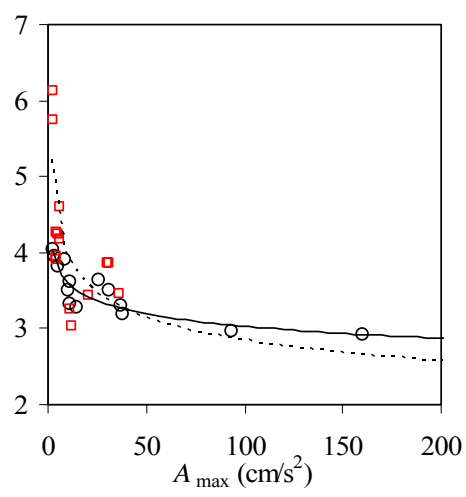

(b)

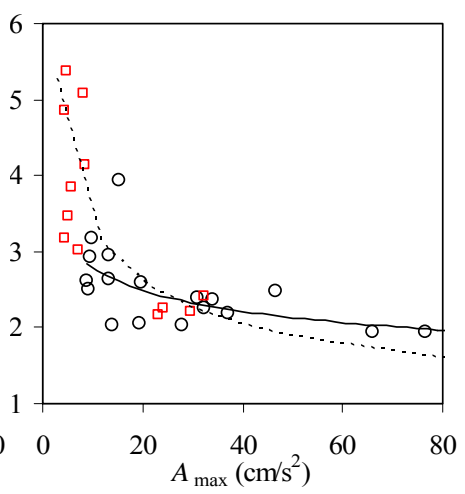

(c)

Figura 4. $\omega$ en función de $A_{\max }$ para los sitios (a) CU, (b) SCT y (c) CD durante sismos de subducción (círculos) y falla normal (cuadrados)

Se ha observado (Reinoso y Ordaz, 1999) que el valor de $T_{s}=0.5$ seg es el límite entre la zona de transición del valle de México y terreno firme, por lo que por convención, se acepta asignar a sitios de terreno firme $T_{\mathrm{s}}=0.5 \mathrm{seg}$; esto es particularmente útil cuando se comparan los parámetros observados en terreno firme y la zona de lago y transición. Se observa que existen diferencias con respecto al origen del sismo (falla normal o subducción) en el patrón de comportamiento del parámetro $\omega$, por lo que sugerimos manejar las bases de datos de manera independiente.

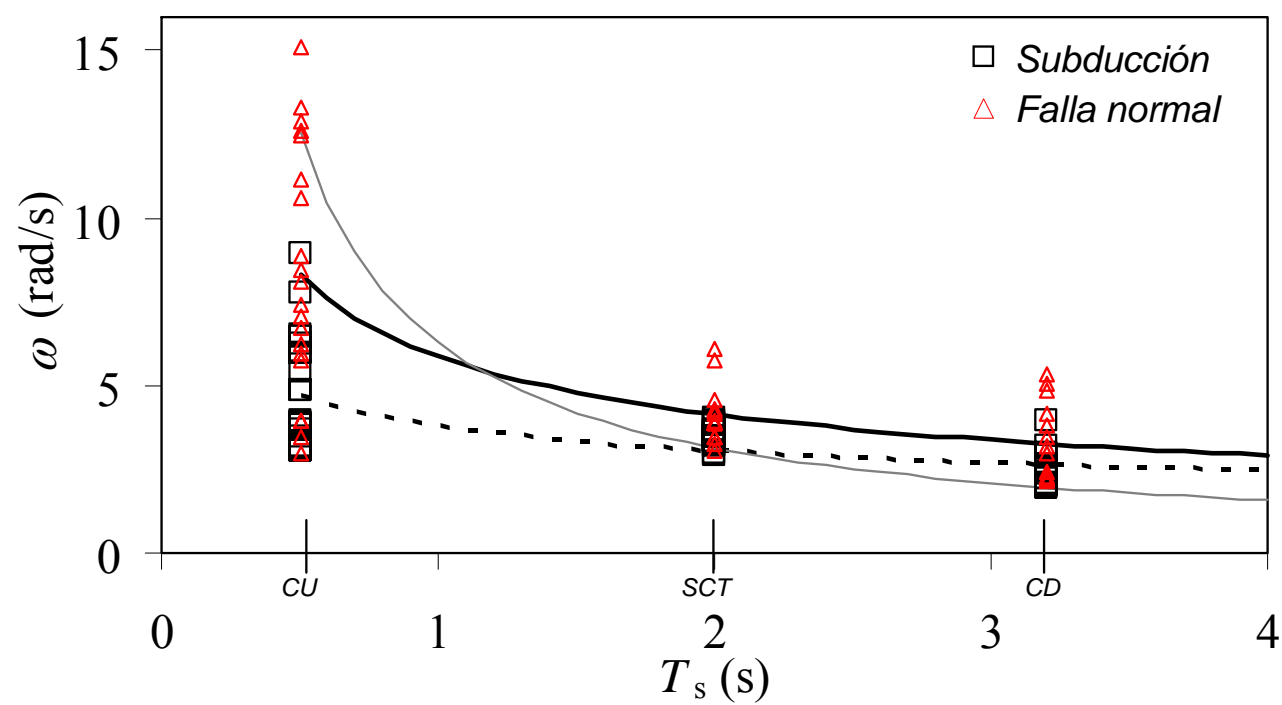

Figura 5. $\omega$ para sismos de subducción (cuadrados) y falla normal (triángulos) y sus tendencias centrales mediante línea discontinua y continua, respectivamente; con línea gris se muestra también la relación obtenida para la frecuencia del suelo $\omega_{\mathrm{s}}=2 \pi / T_{s}$

En la fig. 6 se presenta la dependencia de $\omega$ con respecto al periodo dominante, $T_{s}$ y a la aceleración máxima del suelo, $A_{\max }$, en sitios del valle de México, para sismos de subducción y falla normal, derecha e izquierda, respectivamente, y la superficie que mejor se ajusta. Se asumió que una regresión lineal en el espacio logarítmico es apropiada para describir la dependencia de $\omega$, entonces: 
$\ln \omega=c_{0}+c_{1} \ln T_{s}+c_{2} \ln A_{\max }$

Se encontró que el parámetro $\omega$ para sismos de tipo subducción puede calcularse como

$\ln \omega=1.8349-0.4043 \ln T_{s}-0.1146 \ln A_{\max }$

válida para $T_{\mathrm{s}} \geq 0.5$ (para terreno firme se considera que $T_{s}=0.5 \mathrm{seg}$ ).

y para sismos de falla normal se determinó que

$\ln \omega=1.9628-0.5508 \ln T_{s}-0.0834 \ln A_{\max }$
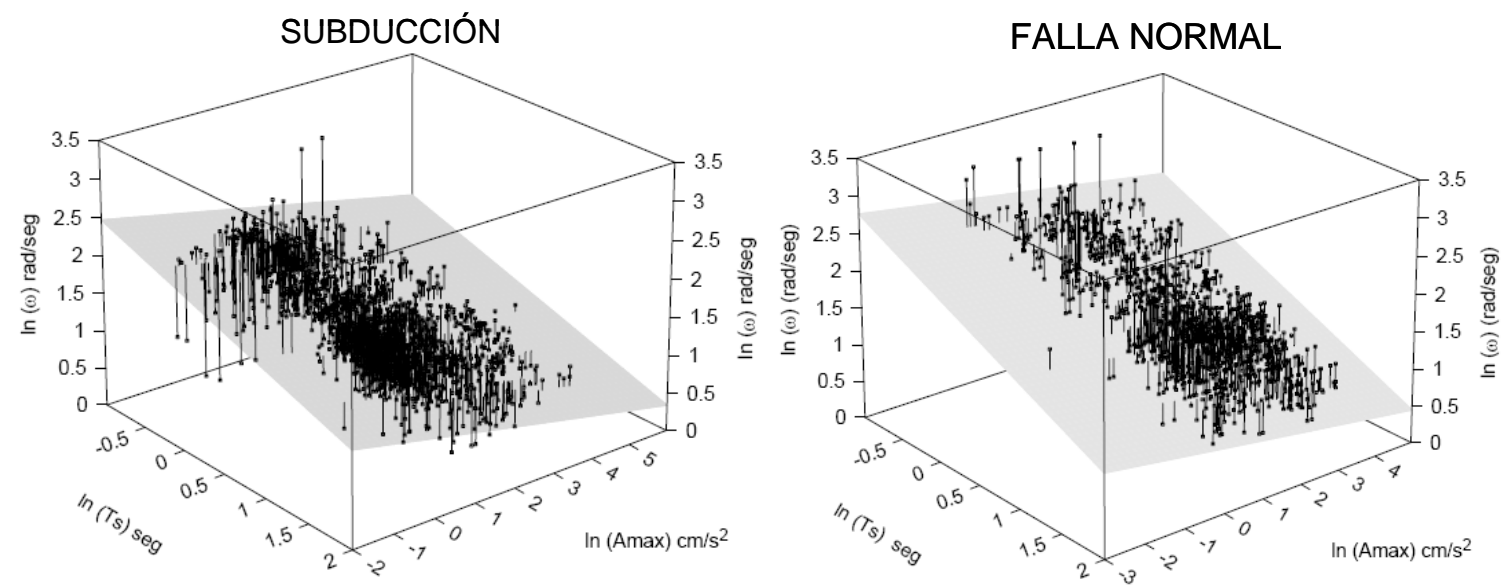

Figura 6. Parámetro $\omega$ en función del periodo del suelo $\left(T_{s}\right)$ y $A_{\max }$ en sitios del valle de México para sismos de subducción (derecha) y falla normal (izquierda)

La estimación del parámetro $\omega$ no está libre de incertidumbres. Estas incertidumbres, como es bien conocido, son factores clave para el análisis de peligro desde un punto de vista probabilista y es comúnmente descrita por la desviación estándar. En la fig. 7a se presenta la frecuencia de $\ln T_{s}$ y en las figs. 7b y 7c la frecuencia de $\ln A_{\max }$ resultados de la muestra considerada para sismos de subducción y falla normal en el valle de México. En estas figuras se presentan los valores de "sigma" para el periodo y aceleración máxima del suelo para sismos de subducción (fig. 7b) y falla normal (fig. 7c). Nótese que la sigma de la muestra para ambos tipos de sismos es la misma $\left(\sigma_{\ln A_{\max }}=0.93\right)$.

Resultado de lo anterior y debido a que $T_{\mathrm{s}}$ y $A_{\max }$ son variables aleatorias independientes, la desviación estándar de $\ln \omega$ puede calcularse como

$\sigma_{\ln \omega} \approx \sqrt{c_{1}^{2} \sigma_{\ln T_{s}}^{2}+c_{2}^{2} \sigma_{\ln A_{\max }^{2}}}$ 


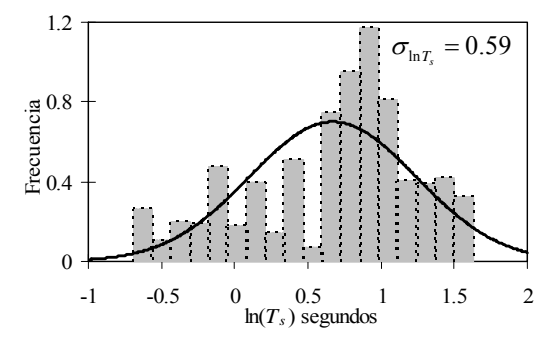

(a)

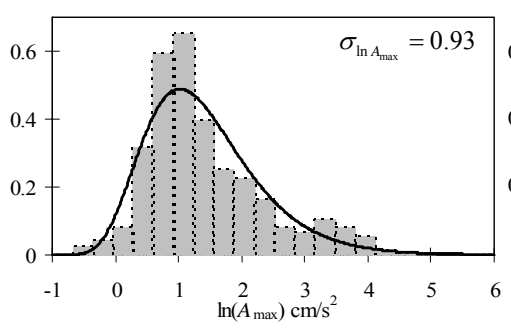

(b)

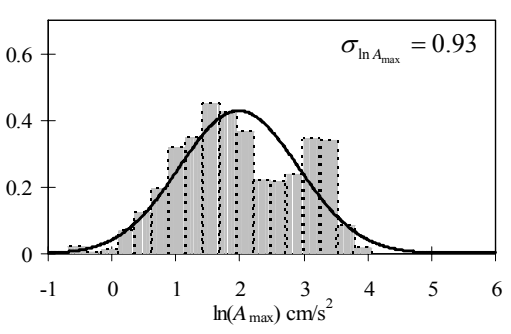

(c)

Figura 7. Frecuencia y desviación estándar para (a) $\ln T_{\mathrm{s}}$, (b) $\ln A_{\max }$ en sitios del valle de México para sismos de subducción y (c) $\ln A_{\max }$ en sitios del valle de México para sismos de falla normal

Del planteamiento anterior, se obtienen valores de $\sigma_{\ln \omega}=0.26$ y $\sigma_{\ln \omega}=0.33$ para sismos de subducción y falla normal, respectivamente.

Lo anterior permite estimar $V_{\max }$ para sitios en el valle de México en función de $A_{\max } \mathrm{y} \omega$; donde obteniendo logaritmo en ambos lados de la ec. 1, con el fin de sustituir el término de las ecs. 4 o 5 , tenemos

$\ln V_{\max }=\ln A_{\max }-\ln \omega$

Sustituyendo la ec. 4 en la ec. 7 se obtiene

$$
\ln V_{\max }=-1.8349+1.1146 \ln A_{\max }+0.4043 \ln T_{s}
$$

donde $V_{\max }$ está función de $A_{\max } \mathrm{y}$ de $T_{s}$ para sismos de tipo subducción e implícitamente del parámetro $\omega$ Sustituyendo la ec. 5 en la ec. 7 se obtiene el equivalente para sismos de falla normal

$$
\ln V_{\max }=-1.9628+1.0834 \ln A_{\max }-0.5508 \ln T_{s}
$$

Las ecuaciones anteriores son validas para $T_{\mathrm{s}} \geq 0.5 \mathrm{seg}$, y para terreno firme $T_{\mathrm{s}}=0.5 \mathrm{seg}$.

Las ecs. 8 ó 9 en conjunto con una ley de atenuación existente de $A_{\max }$ y de un modelo de la actividad sísmica de la fuente, permite realizar un análisis de peligro sísmico probabilista. Sin embargo, para llevar a cabo lo anterior aún es necesario contar con parámetros estadísticos como la desviación estándar del logaritmo natural $\sigma_{\ln V_{\max }}$ y el coeficiente de correlación $\rho_{\ln V_{\max } \ln A_{\max }}$.

Se encontró que $\ln A_{\max }$ y $\ln \omega$ están poco correlacionadas ( $\rho_{\ln A_{\max } \ln \omega}=-0.35$ y -0.30 para sismos de subducción y falla normal, respectivamente), entonces la incertidumbre para $\ln V_{\max }$ puede calcularse como:

$$
\sigma_{\ln V_{\max }} \approx \sqrt{c_{1}^{2} \sigma_{\ln T_{s}}^{2}+\left(1-c_{2}\right)^{2} \sigma_{\ln A_{\max }}^{2}+\sigma_{\ln \omega}^{2}-2\left(1-c_{2}\right) \rho_{\ln A_{\max } \ln \omega} \sigma_{\ln A_{\max } \sigma_{\ln \omega}}}
$$


De lo anterior, se obtuvieron valores de $\sigma_{\ln V_{\max }}=1.18$ y 1.20 , valores mayores que los típicos para leyes de atenuación en terreno duro y firme que están entre 0.6 y 0.8 . Sin embargo, consideramos que estos valores son aceptables con fines de ingeniería dado que la expresión propuesta toma en cuenta todas las incertidumbres de las propiedades locales del suelo de los sitios estudiados. Esto es conocido puesto que el sigma para una estación individual es menor que el sigma encontrado para varias estaciones (Atkinson, 2006).

Los coeficientes de correlación se calcularon comparando la velocidad medida y la calculada (ecs. 8 y 9) resultando en $\rho_{\ln V_{\max } A_{\max }} \approx 0.965$ y 0.957 para sismos de subducción y falla normal, respectivamente. En la fig. 8 se presenta la relación entre $V_{\max }$ calculada (ec. 8 ó 9, subducción y falla normal, respectivamente) y $V_{\max }$ medida en sitios en el valle de México (puntos cuadrados). Se puede apreciar que existe una estimación adecuada de $V_{\max }$ en función de $A_{\max }$ conforme el valor de $V_{\max }$ se incrementa; para valores pequeños de $V_{\max }$ calculada la estimación es menor a la medida.
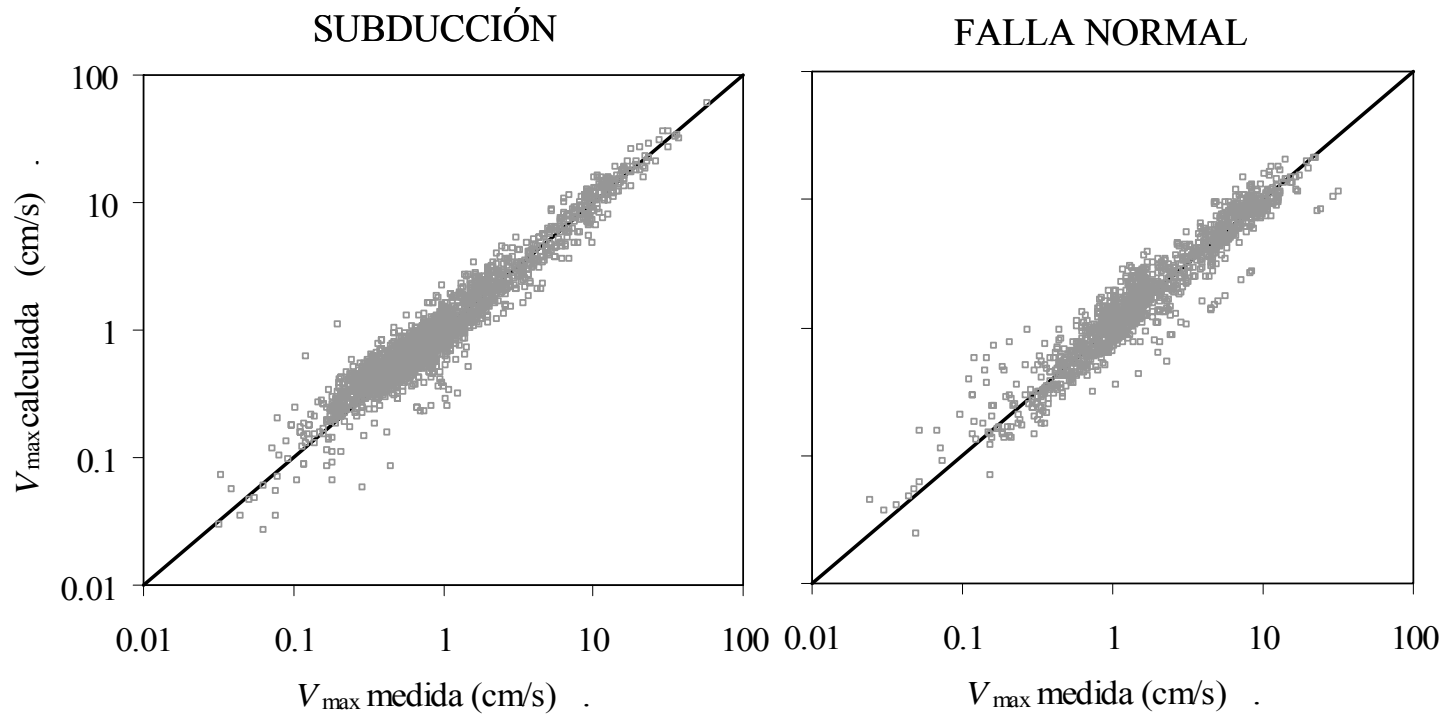

Figura 8. Comparación de $V_{\max }$ medida y $V_{\max }$ calculada mediante las ecs. 8 y 9 (sismos de subducción y falla normal, respectivamente) para sitios dentro del valle de México

\section{ESTIMACIÓN DEL PARÁMETRO $\omega$ PARA SITIOS FUERA DEL VALLE DE MÉXICO}

Para sitios fuera del valle de México se analizaron 54 estaciones en terreno firme, de las cuales se obtuvieron 1612 movimientos registrados en ambas direcciones, norte-sur y este-oeste; 1186 y 426 sismos de subducción y falla normal, respectivamente. Procediendo de manera similar que para sitios en el valle de México se obtuvieron sus intensidades pico $\left(A_{\max }\right.$ y $\left.V_{\max }\right)$ y el parámetro $\omega$.

Se buscó establecer una correlación del parámetro $\omega$ con la intensidad del sismo, como previamente se realizó para sitios en el valle de México; sin embargo, se encontró una gran variabilidad de $\omega \operatorname{con} A_{\max }$. Debido a lo anterior, para sitios localizados fuera del valle de México, se obtuvo un valor promedio del parámetro $\omega$ como se presentan en estudios previos (Newmark y Hall, 1982; Santa-Cruz et 
al., 2000), pero considerando la diferencia del origen del sismo. El valor del logaritmo de $\omega$ calculado fue de 3.27 y 3.07 para sismos de subducción y falla normal, respectivamente; por lo tanto, sustituyendo estos valores en la ec. 7, la expresión de $V_{\max }$ en función de $A_{\max }$ ante eventos de subducción queda como

$\ln V_{\text {max }}=\ln A_{\max }-3.27$

y para eventos de falla normal como

$\ln V_{\max }=\ln A_{\max }-3.07$

Considerando nula la correlación entre $\omega$ y $A_{\max }$, la desviación estándar de $\ln \omega$ puede calcularse como:

$\sigma_{\ln V_{\max }} \approx \sqrt{\sigma_{\ln \omega}^{2}+\sigma_{\ln A_{\max }}^{2}}$

El valor de $\sigma_{\ln A_{\max }}$ puede obtenerse de leyes de atenuación existentes. Considerando las expresiones de Ordaz et al. (1989) y García et al. (2005) se tiene que $\sigma_{\ln A_{\max }}=0.57$ y 0.66 para sismos de subducción y falla normal, respectivamente. Por otro lado, en la fig. 9 se presenta la distribución de la frecuencia de $\ln \omega$ resultado de la muestra considerada para sismos de subducción y falla normal fuera del valle de México. También en la figura se presentan los valores de "sigma" del parámetro $\omega$ para sismos de subducción y falla normal.

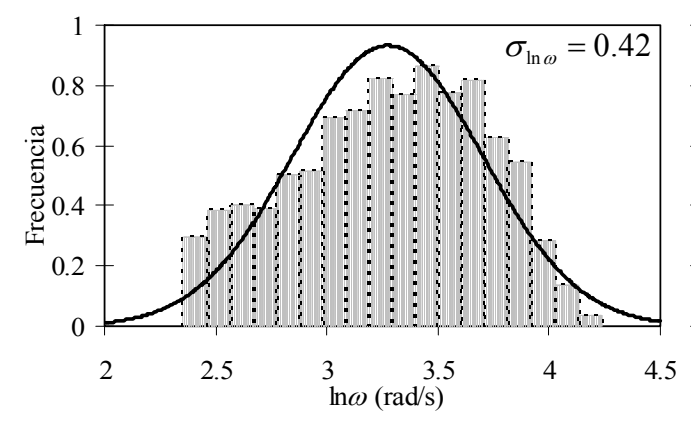

(a)

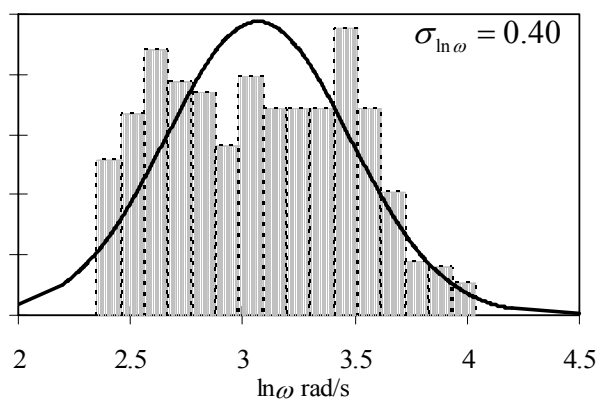

(b)

Figura 9. Frecuencia y desviación estándar para $\ln \omega$ en sitios fuera del valle de México para sismos de (a) subducción y (b) falla normal

A partir de la ec. 13 se obtuvieron valores de $\sigma_{\ln V_{\max }}=0.70$ y 0.77 , valores típicos para leyes de atenuación, y coeficientes de correlación comparando la velocidad medida y calculada (ecs. 11 y 12) de $\rho_{\ln V_{\max } A_{\max }} \approx 0.891$ y 0.829 en cada caso; el primer valor de cada parámetro estadístico es para sismos de subducción y el segundo para eventos de falla normal. En la fig. 10 se presenta lo mismo que la fig. 8 pero para sitios fuera del valle de México. Nótese que los valores de correlación $\rho_{\ln V_{\max } A_{\max }}$ en sitios fuera del valle de México son menores en comparación con la obtenida para los sitios en el valle de México (ver fig. 8). 

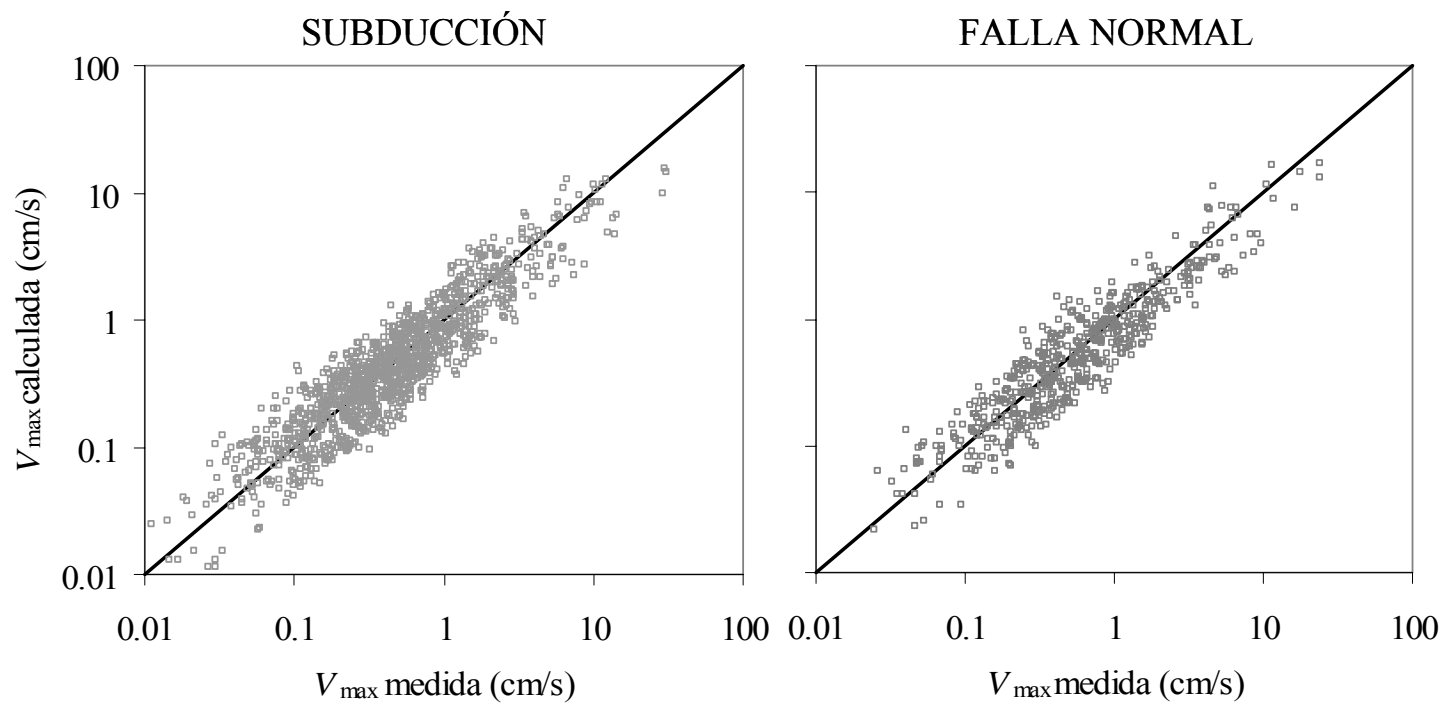

Figura 10. Comparación de $V_{\max }$ medida y $V_{\max }$ calculada mediante las ecs. 11 y 12 (sismos de subducción y falla normal, respectivamente) para sitios fuera del valle de México

\section{EJEMPLO DE APLICACIÓN: ANÁLISIS PROBABILISTICO DEL PELIGRO SÍSMICO DE CONTENIDOS Y ELEMENTOS NO ESTRUCTURALES}

La ec. 14 (Esteva, 1967; Cornell, 1968) presenta la manera de obtener la tasa de excedencia de valores de la aceleración máxima del suelo:

$v(a)=\sum_{i=1}^{N} \int_{M_{O}}^{M_{U}} \lambda_{o} p(M) \cdot \operatorname{Pr}(A>a \mid M, R) d M$

En la ec. 14, $N$ es la número total de fuentes sísmicas, $M_{O}$ y $M_{U}$ son la magnitud mínima y máxima que se puede generar en la fuente sísmica; $\lambda_{o}$ es la tasa de excedencia de magnitud de $M=M_{O}, p(M)$ es la función de densidad de probabilidad de la magnitud, $\operatorname{Pr}(A>a \mid \mathrm{M}, R)$ es la probabilidad que la aceleración máxima del terreno $A$ exceda el valor de $a$ en el sitio, dado que sucedió un sismo de magnitud $M$ a la distancia $R$.

Sin embargo, en el caso de la estimación probabilista del peligro sísmico asociado a dos parámetros correlacionados, la tasa de excedencia se puede calcular de acuerdo a lo propuesto por Santa Cruz et al. (2000) como

$$
v(a, v)=v(a)+v(v)-\sum_{i=1}^{N} \int_{M_{O}}^{M_{U}} \lambda_{o} p(M) \cdot \operatorname{Pr}(A>a \bigcup V>v \mid M, R) d M
$$

donde $v(a)$ y $v(v)$ son las tasas de excedencia de aceleraciones y velocidades, respectivamente. 
Para evaluar los términos $v(v)$ y $\lambda_{o} p(M) \cdot \operatorname{Pr}(A>a \bigcup V>v \mid M, R)$ de la ec. 15 se requiere contar no sólo con un modelo de la actividad sísmica y de una la ley de atenuación de $V_{\max }$, sino además se necesita conocer la distribución conjunta de $A$ y $V$. La obtención de $V_{\max }$ en función de $A_{\max }$ y la correlación entre las variables aleatorias $A$ y $V$ se estima como discutió previamente, lo que permite más tarde calcular el análisis probabilista de peligro sísmico bivariado.

Presentamos un ejemplo del cálculo del peligro sísmico probabilista bivariado para dos sitios localizados fuera del valle de México, afectado por una fuente sísmica puntual; se considera cada sitio localizados a 30 y $100 \mathrm{~km}$, respectivamente. El modelo de sismicidad considerado para estimar la actividad sísmica en la fuente es el dado por Cornell and Varmacke (1969), definido como

$$
\lambda(M)=\left\{\begin{array}{lll}
\lambda_{o} \frac{\exp (-\beta M)-\exp \left(-\beta M_{U}\right)}{\exp \left(-\beta M_{O}\right)-\exp \left(-\beta M_{U}\right)} & \text { si } & M_{O} \leq M<M_{U} \\
0 & \text { si } & M \geq M_{U}
\end{array}\right.
$$

donde $\lambda(M)$ es la tasa de excedencia de magnitud de la fuente sísmica, $M_{O}$ y $M_{U}$ son los valores de magnitud mínima y máxima, respectivamente; $\lambda_{o}$ es la tasa de excedencia de magnitud para $M=M_{O} \mathrm{y}$ $\beta$ es un valor esperado.

Entonces, la función de densidad de probabilidad de la magnitud, $p(M)$, se relaciona con la tasa de excedencia de magnitud, tomando la forma

$$
p(M)=\left\{\begin{array}{lll}
\frac{\beta \exp (-\beta M)}{\exp \left(-\beta M_{O}\right)-\exp \left(-\beta M_{U}\right)} & \text { si } & M_{O} \leq M<M_{U} \\
0 & \text { si } & M \geq M_{U}
\end{array}\right.
$$

Para fines de ejemplificar la obtención de curva de peligro sísmico bivariada $v(a, v)$, los parámetros de la sismicidad usados son: $\lambda_{o}=1 /$ año, $\beta=2, M_{O}=2$ y $M_{U}=8$ (Ordaz, 2004). Asimismo, la forma de la ley de atenuación y sus respectivos coeficientes que relacionan la magnitud y distancia de la fuente al sitio con $A_{\max }$ son tomados del trabajo de Ordaz (2004). La desviación estándar del logaritmo natural de $A_{\max }$ se consideró igual a 0.57 .

En la fig. 11 se presenta la curva de peligro sísmico bivariada para los dos sitios desplantados en terreno firme a $30 \mathrm{~km}$ (fig. 11a) y $100 \mathrm{~km}$ (fig. 11b) de la fuente sísmica, respectivamente. Cada una de las curvas de peligro es resultado de aplicar la ec. 15 donde se utilizan los parámetros sísmicos y de atenuación de $A_{\max }$ antes señalados. Asimismo, se consideró que en la fuente se originan sismos de tipo subducción por lo que se utilizó la ec. 11 y sus correspondientes valores estadísticos. De la figura se puede observar que conforme la fuente sísmica se encuentra más alejada $(R=100 \mathrm{~km})$ del sitio de interés, la tasa de excedencia sísmica es menor y por lo tanto la probabilidad de que mayor número de contenidos colapsen, se reduce.

Este tipo de curvas de peligro bivariadas calculadas podría más tarde usarse en la estimación de la pérdida esperadas para tuberías enterradas, contenidos y elementos no estructurales. 


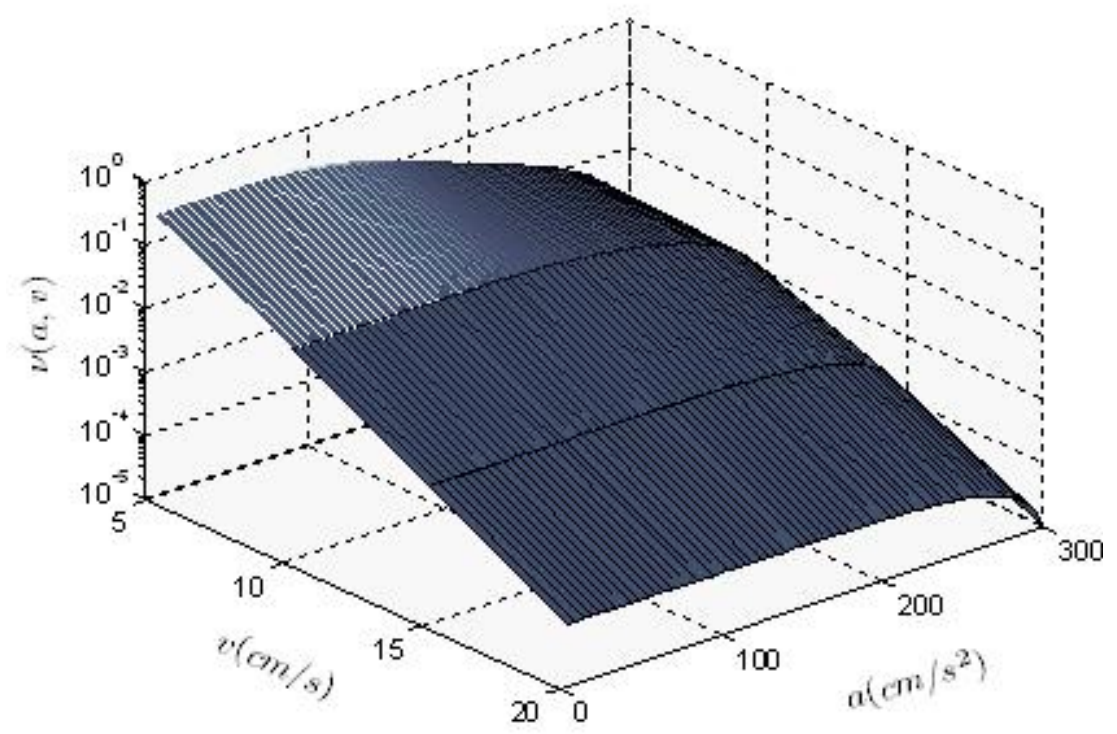

(a)

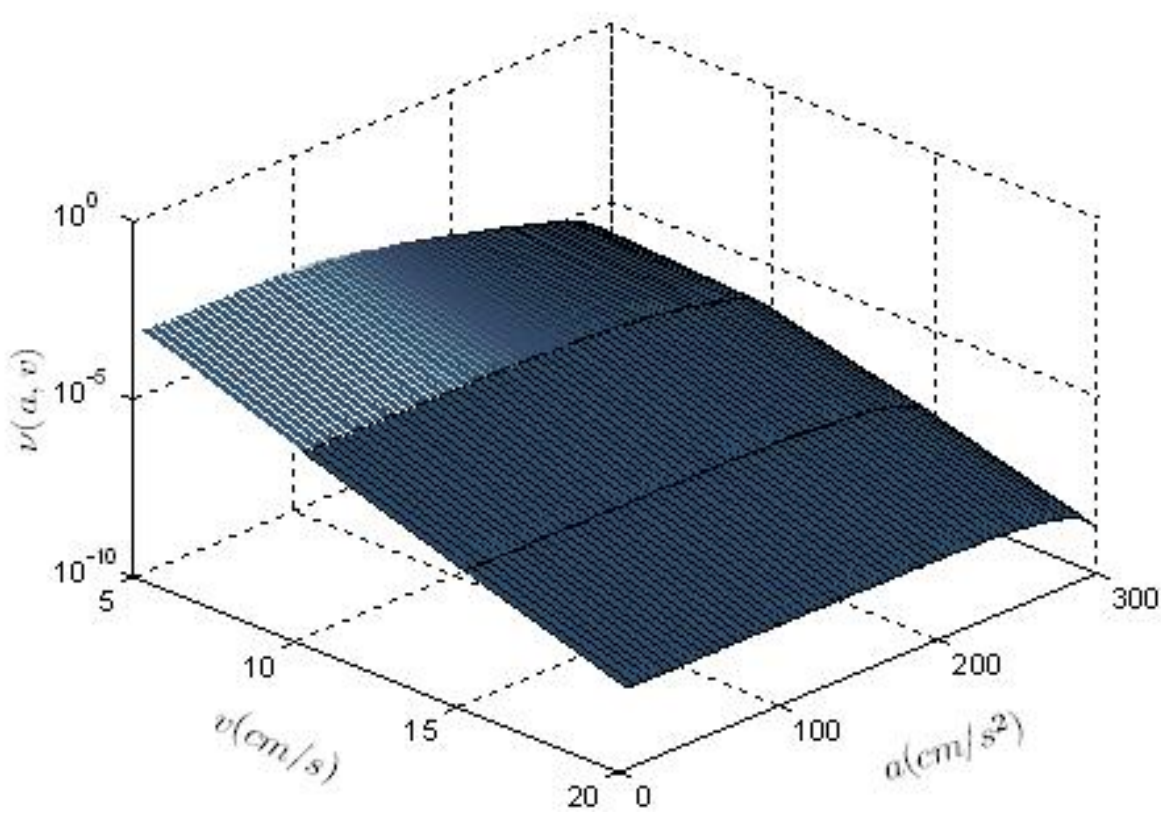

(b)

Figura 11. Curva de peligro sísmico bivariada de $a, v, v(a, v)$, calculada con la ec. 15. Esta curva corresponde a una fuente puntual donde se originan sismos de tipo subducción y una ley de atenuación probabilista, y un error en la incertidumbre en la ley de atenuación de aceleración de $\sigma=0.57$. a) $R=30$ $\mathrm{km}$ y b) $100 \mathrm{~km}$ 


\section{CONCLUSIONES}

Se presenta una expresión que correlaciona los parámetros sísmicos de aceleración $\left(A_{\max }\right)$ y velocidad $\left(V_{\max }\right)$ máximas del suelo que son usados frecuentemente en la estimación de daño en tuberías enterradas, contenidos y elementos no estructurales. Esta expresión permite estimar el valor del parámetro $V_{\max }$ en función de $A_{\max }$, por lo que, de una ley de atenuación existente para $A_{\max } \mathrm{y}$ de un modelo de la actividad sísmica de la fuente, es posible realizar un análisis probabilista del peligro sísmico bivariado, que resulta útil en la estimación de pérdidas en contenidos y elementos no estructurales.

Se obtuvieron valores de errores para sitios en el valle de México encontrando una desviación estándar condicional del logaritmo natural de 1.18 y 1.20 y un coeficiente de correlación de $\rho_{\ln V_{\max } A_{\max }}=0.965$ y 0.957 para sismos de subducción y falla normal, respectivamente, útil para fines prácticos debido a que la expresión propuesta toma en cuenta las incertidumbres de las propiedades locales del suelo de los sitios estudiados del valle de México. Por otro lado, para sitios de terreno firme fuera del valle de México se encontraron valores de desviación estándar menores a los sitios en el valle de México y coeficientes de correlación mayores a 0.8 .

Por último, se presenta un ejemplo que ilustra la curva de peligro sísmico expresada en términos de la aceleración y velocidad máxima del suelo para dos sitios de terreno firme. Estas curvas de peligro bivariadas calculadas podría usarse en la estimación de la pérdida esperadas para tuberías enterradas, contenidos y elementos no estructurales.

\section{AGRADECIMIENTOS}

Los autores agradecen las observaciones por parte de los revisores anónimos que enriquecieron el artículo.

\section{REFERENCIAS}

Abrahamson, N y W Silva (2008), "Summary of the Abrahamson \& Silva NGA ground-motion relations", Vol. 24, No. 1, pp. 67-97.

Akkar, S y J Bommer (2007), "Empirical Prediction Equations for Peak Ground Velocity Derived from Strong-Motion Records from Europe and the Middle East", Bulletin Seismological Society of America, Vol. 97, pp. 511-530.

Al Abadi, A, N Lam y E Gad (2006), "A simple displacement-based model for predicting seismically induced overturning”, Journal of Earthquake Engineering, Vol. 10, No. 6, pp. 775-814.

Ambraseys, N (2006), "The prediction of earthquake peak ground acceleration in Europe", Earthquake Engineering and Structural Dynamics, Vol. 24, No. 4, pp. 467-490.

Arredondo, C y E Reinoso (2008), "Influence of frequency content and peak intensities in the rocking seismic response of rigid bodies", Journal of Earthquake Engineering, Vol. 12, No. 4, pp. 517-533.

Atkinson, G M (2006), "Single-Station Sigma”, Bulletin Seismological Society of America, Vol. 96, No. 2, pp. 446-455.

Baker, J y N Jarayam (2008), "Correlation of spectral acceleration values from NGA ground motion models", Vol. 24, No. 1, pp. 299-317. 
Boore, D y G Atkinson, (2008), "Ground-motion prediction equations for the average horizontal component of PGA, PGV, and 5\%-damped PSA at spectral periods between $0.01 \mathrm{~s}$ and $10.0 \mathrm{~s}$ ", Vol. 24, No. 1, pp. 99-138.

Campbell, K y Y Bozorgnia (2008), "NGA ground motion model for the geometric mean horizontal component of PGA, PGV, PGD and 5\% damped linear elastic response spectra for periods ranging from 0.01 and 10 s", Vol. 24, No. 1, pp. 139-171.

Chiou, B y R Youngs (2008), “An NGA model for the average horizontal component of peak ground motion and response spectra", Vol. 24, No. 1, pp. 173-215.

Cornell, C A (1968), "Engineering seismic risk analysis", Bulletin Seismological Society of America, Vol. 58 , No. 5 , pp. 1583-1606.

Cornell, C A y E Vanmarcke (1969), "The mayor influences on seismic risk", Proc. 4th World Conference on Earthquake Engineering, Santiago de Chile, 15-19 July.

Choi, B y D Tung (2002), "Estimating sliding displacement of an unanchored body subjet to earthquake exitation", Earthquake Spectra, Vol. 18, No. 4, pp. 601-613.

Esteva, L (1967). "Criterios para la construcción de espectros para diseño sísmico", 3er Simposio Panamericano de Estructuras, Caracas, Venezuela.

Fajfar, P, T Vidic, y M Fischinger (1992), "On energy demand and supply in SDOF systems", Nonlinear Sesismic Analysis and Design of Reinforced Concrete Buildings, P. Fajfar y H. Krawinkler, editors, Elsevier.

García, D, S K Singh, M Herráiz, M Ordaz y J F Pacheco (2005), "Inslab Earthquakes of Central Mexico: Peak Ground-Motion Parameters and Response Spectra", Bulletin Seismological Society of America, Vol. 95, No. 6, pp. 2272-2282.

Housner, G W y P C Jennings (1982), "Earthquake Design Criteria", EERI Monograph Series, Earthquake Engineering Research Institute, Oakland, C.A.

Ishiyama, Y (1982), "Motions of rigid bodies and criteria for overturning by earthquake excitation", Earthquake Engineering and Structural Dynamics, No. 10, pp. 635-652.

Ishiyama, Y (1984), "Motions of rigid bodies and criteria for overturning by earthquake excitation", Bulletin of the New Zealand Society for Earthquake Engineering, No. 17, 1, pp. 24-37.

Katayama, T, K Kubo y N Sato (1975), "Earthquake damage to water and gas distribution systems", Proceedings of the U.S. National Conference on Earthquake Engineering, Ann Arbor, Michigan, pp. 396405.

Konstantinidis, D y N Makris (2003), "Experimental and analytical studies on the seismic response of slender laboratory equipment", Proceeding of seminar on seismic design, Performance and retrofit of nonstructural components in critical facilities, ATC-29-2, pp. 399-411.

Konstantinidis, D y N Makris (2006), "Experimental and analytical studies on the seismic response of freestanding and restrained laboratory equipment", Proceeding of the 8th national conference on earthquake engineering, San Francisco, California, Paper No. 1619, pp. 18-22.

Newmark, N M y W J Hall (1982), "Earthquake Spectra and Design”, EERI Monograph Series, EERI, Oakland, California.

Ordaz, M, J M Jara y S K Singh (1989), "Riesgo sísmico y espectros de diseño en el estado de Guerrero", VIII Congreso Nacional Ingeniería Sísmica, Acapulco, México, D40-D56. 
Ordaz, M y S K Singh (1992), "Source spectra and spectral attenuation of seismic waves from Mexican earthquakes, and evidences of amplification in the hill zone of Mexico City", Bulletin Seismological Society of America, Vol. 82, No. 1, pp. 24-43.

Ordaz, M (2004), "Some integrals useful in Probabilistic Seismic Hazard Assessment", Bulletin Seismological Society of America, Vol. 94, No. 4, pp. 1510-1516.

O’Rourke, M y G Ayala (1993), "Pipeline Damage to Wave Propagation", Journal of Geotechnical Engineering, ASCE. Vol. 119, No. 9.

Oppenheim, A y R Schafer (1975), "Digital signal processing”, Prentice-Hall.

Pineda, O y M Ordaz (2004), "Mapas de velocidad máxima del suelo para la Ciudad de México", Revista de Ingeniería Sísmica, Sociedad Mexicana de Ingeniería Sísmica, No. 71, pp. 37-62.

Pineda, O (2006), "Estimación de daño sísmico en tuberías enterradas", Tesis de doctorado, Posgrado de Ingeniería, UNAM, pp. 1-102.

Psycharis, N, C Syngros, P Mimoglou y I Taflambas (2002), "Parametric investigation of the overturning of rigid blocks under dynamic loading", 12th European Conference on Earthquake Engineering, London, U.K., 0.13, September.

Rathje, E M, N A Abrahamson y J D Bray (1998), "Simplified frequency content estimates of earthquake ground motions", J. Geotech. Eng. Div., Am. Soc. Civ. Eng. Vol. 124, No. 2, 150-159.

Rathje, E M, F Farai, S Russell, y J D Bray (2004), "Empirical Relationships for frequency Content Parameters of Earthquake Ground Motions", Earthquake Spectra, Vol. 20, No. 1, pp. 119-144.

Reinoso, E y M Ordaz (1999), "Spectral ratio for Mexico city from free-field recordings", Earthquake Spectra, Vol, 15, No. 2, pp. 273-295.

Ridell, R (2007), “On Ground motion intensity indices”, Earthquake Spectra, Vol. 23, No. 1, pp. 147-173.

Santa-Cruz, S, M Ordaz y R Guerrero (2000), "Estimación de perdidas en contenidos dentro de naves industriales debido a sismos", XII Congreso Nacional de Ingeniería estructural, León, Gto. México, Noviembre.

Tso, W, T Zhu y A Heidebrecht (1992), "Engineering implication of ground motion A/V ratio", Soil Dynamics and Earthquake Engineering, Vol. 11, No. 3, pp. 133-144. 